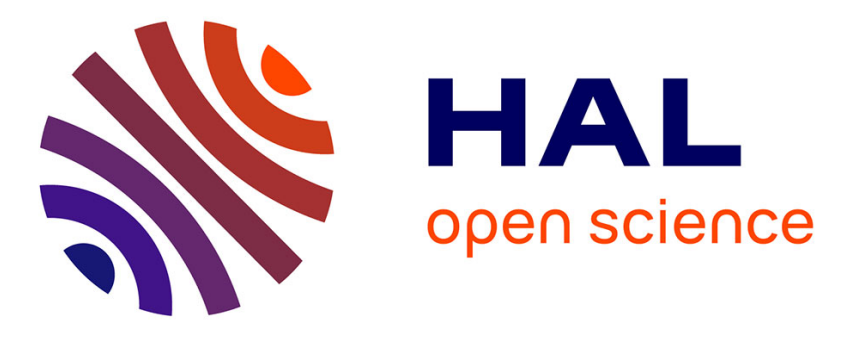

\title{
Emergence of rogue waves from optical turbulence
}

\author{
Kamal Hammani, Bertrand Kibler, Christophe Finot, Antonio Picozzi
}

\section{To cite this version:}

Kamal Hammani, Bertrand Kibler, Christophe Finot, Antonio Picozzi. Emergence of rogue waves from optical turbulence. Physics Letters A, 2010, 374 (34), pp.3585-3589. 10.1016/j.physleta.2010.06.035 . hal-00491491

\section{HAL Id: hal-00491491 \\ https://hal.science/hal-00491491}

Submitted on 11 Jun 2010

HAL is a multi-disciplinary open access archive for the deposit and dissemination of scientific research documents, whether they are published or not. The documents may come from teaching and research institutions in France or abroad, or from public or private research centers.
L'archive ouverte pluridisciplinaire HAL, est destinée au dépôt et à la diffusion de documents scientifiques de niveau recherche, publiés ou non, émanant des établissements d'enseignement et de recherche français ou étrangers, des laboratoires publics ou privés. 


\title{
Emergence of rogue waves from optical turbulence
}

\author{
Kamal Hammani, Bertrand Kibler,* Christophe Finot, and Antonio Picozzi \\ Laboratoire Interdisciplinaire Carnot de Bourgogne, UMR 5209 CNRS-Université de \\ Bourgogne, 9 Avenue Alain Savary, 21000 Dijon, France
}

*Corresponding author: Bertrand.Kibler@u-bourgogne.fr

Laboratoire Interdisciplinaire Carnot de Bourgogne,

UMR 5209 CNRS-Université de Bourgogne, 9 Avenue Alain Savary, 21000 Dijon, France

$$
\begin{gathered}
\text { Phone: }+33(0) 380395932 \\
\text { Fax : +33(0)3 } 80395971
\end{gathered}
$$

\begin{abstract}
We provide some general physical insights into the emergence of rogue wave events from optical turbulence by analyzing the long term evolution of the field. Depending on the amount of incoherence in the system (i.e., Hamiltonian), we identify three turbulent regimes that lead to the emergence of specific rogue wave events: (i) persistent and coherent rogue quasi-solitons, (ii) intermittent-like rogue quasi-solitons that appear and disappear erratically, and (iii) sporadic rogue waves events that emerge from turbulent fluctuations as bursts of light or intense flashes.
\end{abstract}

Keywords: Nonlinear optics; Pulse propagation and temporal solitons; Coherence; Statistical optics; Optical turbulence 


\section{Introduction}

The first observation of statistical characteristics analogous to the hydrodynamic rogue waves in nonlinear optical fiber-based systems has been reported recently by Solli et al. in 2007 [1]. However, many aspects of these noise-induced fluctuations in supercontinuum generation experiments and subsequent dynamics leading to high amplitude "optical rogue waves" can be retrieved in the pioneer work of Islam et al. in 1989 and in more recent works [2-6]. In this context, there has been a tremendous activity devoted to the identification and the understanding of the mechanisms underlying the intricate process of optical rogue wave generation. By analogy with hydrodynamic systems [7], the recent studies in optics have confirmed the major role of the interplay between linear and nonlinear effects in the formation of L-shaped distributions of the optical intensities, such as, e.g., the convective effect due to third order dispersion, the modulation instability, or the collision of solitons or breathers structures $[1,8-19]$. These previous works have been focused on the study of two well-known classes of solutions of the NonLinear Schrödinger Equation (NLSE), namely soliton or quasi-soliton solutions and, on the other hand, Akhmediev Breathers $(\mathrm{AB})$ solutions, which are essentially relevant to the integrable limit of the NLSE. In this way, the transition from the dynamics of AB to 'rogue solitons' has been assumed by introducing specific perturbations to the NLSE $[18,20]$. It turns out that, as a rather general rule, the collision of (quasi-)solitons or the collision of $A B$, have been recognized as the essential mechanisms responsible for the generation of optical rogue waves [1,8-9,11-19].

In this Letter we propose a novel approach for the understanding of the generation of optical rogue waves. Our approach is based on the study of the impact of the amount of incoherence in the nonlinear system. More specifically, we identify three turbulent regimes that lead to the emergence of specific extreme wave characteristics. The first regime (i) has been 
studied in various circumstances and refers to '(quasi-)solitonic turbulence' [21,22]. Its long term dynamics is characterized by a robust and persistent coherent rogue (quasi-)soliton that propagates in the midst of small-scale fluctuations. The second regime (ii) is characterized by intermittent-like rogue (quasi-)soliton structures, which appear and disappear erratically from turbulent fluctuations. The third regime (iii) has no relation with (quasi-)solitons and refers to sporadic rogue wave events that emerge from a fully turbulent state: they manifest themselves by means of bursts of light or intense 'flashes' during the propagation.

We consider the one-dimensional NLSE in the presence of Third-Order Dispersion (TOD), which has been shown to lead to the generation of optical rogue events $[18,19]$ :

$$
i \frac{\partial u}{\partial z}=-\frac{1}{2} \frac{\partial^{2} u}{\partial t^{2}}+i \sigma \frac{\partial^{3} u}{\partial t^{3}}-|u|^{2} u
$$

For convenience, we normalized the problem with respect to the nonlinear length $\mathrm{L}_{0}=1 / \gamma \mathrm{P}$ and time $\tau_{0}=\left(\beta_{2} \mathrm{~L}_{0}\right)^{1 / 2}$, where $\gamma$ is the nonlinear coefficient, $\mathrm{P}$ the average power of the field and $\beta_{2}$ the second-order dispersion coefficient. In these units, the normalized TOD coefficient reads $\sigma=\beta_{3} /\left(6 \mathrm{~L}_{0}^{1 / 2} \beta_{2}^{3 / 2}\right), \beta_{3}$ being the TOD coefficient. The NLSE conserves three important quantities, the normalized power $N=\int|u(t)|^{2} d t$, the momentum $M=\int \omega|u(\omega)|^{2} d \omega$ and the 'energy' (Hamiltonian) $\mathrm{H}=\mathrm{H}_{\mathrm{L}}+\mathrm{H}_{\mathrm{NL}}$, which has a linear (dispersive) kinetic contribution $\mathrm{H}_{\mathrm{L}}=$ $\int \mathrm{k}(\omega)|\mathrm{u}(\omega)|^{2} \mathrm{~d} \omega$ and a nonlinear contribution $\mathrm{H}_{\mathrm{NL}}=-1 / 2 \int|\mathrm{u}(\mathrm{t})|^{4} \mathrm{dt}$, where $\mathrm{k}(\omega)=\omega^{2} / 2-\sigma \omega^{3}$ is the linear dispersion relation. The system is said to be 'highly nonlinear' ('weakly nonlinear') when $\varepsilon=\left|\mathrm{H}_{\mathrm{NL}}\right| /\left|\mathrm{H}_{\mathrm{L}}\right| » 1(\varepsilon \ll 1)[21]$. The TOD term in Eq. $(1)(\sigma \neq 0)$ is known to break the integrability of the scalar NLSE. However, Eq.(1) admits quasi-soliton solutions: as a result of the TOD term, the standard soliton solution slowly loses its power through a process analogous to Cherenkov radiation $[21,23]$. 
In the context of '(quasi-)soliton turbulence' [21,22], a nonintegrable Hamiltonian wave system exhibits a thermalization process characterized by an irreversible evolution of the wave towards a specific equilibrium state, in which a (quasi-)soliton structure remains immersed in a sea of small scale fluctuations. This process may be interpreted as a consequence of the fact that soliton collisions in non-integrable systems are inelastic: the big soliton becomes bigger while the weaker one loses its power. The resulting big (quasi-)soliton may thus be regarded as a 'statistical attractor' for the system, while the small-scale fluctuations contain, in principle, all information necessary for a reversible evolution of the system [21,22,24]. Note that this phenomenon of 'energy localization' has been also extensively studied in discrete nonlinear systems [25-29], in particular in relation with the Fermi-Pasta-Ulam problem [30].

However, we underline that, in general, the formation of a large scale coherent structure is only possible if the amount of incoherence in the system is not too large [31-32], a feature that was also observed in the highly incoherent regime of supercontinuum generation [33-35]. This aspect has been the subject of a detailed study in the context of wave condensation, where it was shown that the emergence of a large-scale coherent structure (a plane wave) only occurs below some critical 'energy' [36-38]. The 'energy' refers here to the Hamiltonian, which is known to provide a measure of the amount of incoherence in the system [39]. Inspired from these previous works on wave condensation, we study here the transition from the coherent quasi-soliton regime to the fully turbulent regime by increasing the energy $\mathrm{H}$ of the system. To our knowledge, it is the first time that a condensation-like process is studied with quasi-soliton coherent structures.

We performed intensive numerical simulations of Eq.(1) with periodic boundary conditions. Contrary to previous studies [1,8-19], we are interested here in the long term evolution of the system (typically $\mathrm{z} \sim 2000 \mathrm{~L}_{0}$ ), in which the transient processes associated to 
(multiple) quasi-soliton collisions have died out [40]. Note that light propagation over several thousands of nonlinear lengths $\mathrm{L}_{0}$, is experimentally accessible by using highly nonlinear fibers. Figure 1a reports the average of the maximum intensity peak detected in the temporal window in the last stage of propagation (i.e., over $500 \mathrm{~L}_{0}$ ) as a function of the energy density $\overline{\mathrm{H}}=\mathrm{H} / \mathrm{T}$ (see the caption of Fig. 1). We first remark that, in analogy with wave condensation, there exists a threshold value for the energy, $\overline{\mathrm{H}}_{\mathrm{c}} \sim 15$, above which quasi-soliton structures are no longer generated. In a loose sense, this means that the system is too incoherent ('too hot') to generate a coherent structure when $\overline{\mathrm{H}}>\overline{\mathrm{H}}_{\mathrm{c}}$. We remark that this condensation curve has been obtained by varying the energy $\mathrm{H}$, keeping constant the power $\mathrm{N}$. Note however that a condensation curve may also be retrieved by varying the power $\mathrm{N}$, keeping constant the energy $\mathrm{H}$. In this case, condensation does not take place for a small power N, i.e., there exists a threshold value for the power, $\overline{\mathrm{N}}_{c}$, above which condensation arises. This is in complete analogy with the genuine quantum condensation that occurs in Bose gases.

More precisely, we identified in Fig. 1a three different regimes: For $\overline{\mathrm{H}} \leq 5$, we recover the quasi-soliton turbulent regime (i): a large amplitude quasi-soliton immersed in a sea of smallscale fluctuations eventually emerges from multiple inelastic collisions. Figure $2 \mathrm{a}$ represents the space-time intensity pattern over the last 10 nonlinear lengths. The corresponding Probability Distribution Functions (PDF) of the intensity of the field, and of the maxima of the intensity, are reported in Fig. 2b. They have been detected over the last stage of propagation, i.e., over $500 \mathrm{~L}_{0}$. We point out that, because of the large localization of the power in the quasi-soliton, the field statistics deviates substantially from the Gaussian statistics, i.e. $\mathrm{f}_{\mathrm{I}}(\mathrm{I})=\exp (-\mathrm{I})$ with our normalized units. This observation is corroborated by the high value of $\varepsilon(\sim 3)$ and by the PDF of the intensity maxima, which is centered at a high value $(\sim 30)$ and exhibits a narrow width and symmetric shape, thus confirming the persistence of the high power quasi-soliton structure. 
In the example of Fig. 2 we started the numerical simulation from the fundamental soliton solution $(\sigma=0)$ that has been perturbed by a small noise. However, we underline that the initial condition is not essential: The same regime (i) could be reached starting the simulation from a continuous wave (with the corresponding Hamiltonian $\overline{\mathrm{H}}=-1 / 2$ ), or from a broad initial pulse. These quasi-continuous waves are modulationally unstable and thus rapidly evolve toward a periodic train of quasi-solitons, whose subsequent inelastic collisions eventually bring the system into the persistent and coherent quasi-soliton regime described above through Fig. 2.

In spite of its robustness, the large amplitude quasi-soliton experiences a weak interaction with the small-scale fluctuations in which it propagates, as shown by two successive temporal intensity profiles in Fig. 2(c-d). This interaction becomes stronger as the 'level' of the turbulent fluctuations increases, i.e. as the amount of energy $\mathrm{H}$ in the system increases. Typically, for $5 \leq$ $\overline{\mathrm{H}} \leq 15$, the system enters into the second regime (ii), in which the quasi-soliton structures exhibit a kind of intermittent dynamics. This is illustrated in Figs. 3c-d, which report two successive temporal profiles of the field intensity: the quasi-soliton appears and disappears erratically during the propagation. It is interesting to note that, despite such intermittent-like behavior, the trajectory of the quasi-soliton may still be identified in the space-time intensity pattern (see Fig. 3a). As a result of its interaction with the turbulent fluctuations, the 'lifetime' of the quasi-soliton thus seems to fluctuate significantly. We note that, as for the regime (i), the field does not exhibit a Gaussian statistics (see Fig. 3b). However, the PDF of the maxima of the intensity gets relatively broader and asymmetric with respect to that discussed in regime (i) (see the inset of Fig. 3b). This is due to the intermittent (non-persistent) character of the quasi-soliton structure, whose significant fluctuations tend to favor the high intensity tail of the PDF. The resulting asymmetry in the PDF bears a strong resemblance with the typical L-shaped probability distributions that characterize freak-wave extreme events. 
Finally, for $\overline{\mathrm{H}} \geq 15$ the system enters into the fully incoherent and weakly nonlinear regime of propagation (iii). In this regime the optical field is expected to exhibit a thermalization process toward the equilibrium state [22,41,42], a feature that has been analyzed in various circumstances with optical waves [43-48]. However the analysis of this regime from the point of view of extreme statistics in the spatio-temporal domain has not been the subject of a detailed investigation. The corresponding spatiotemporal intensity pattern reveals that very short-lived rogue wave events may still emerge from the turbulent field, although these extreme events become rare (see Fig. 4a). The phenomenology of these events may resemble the extreme waves reported in Ref.[49] in optical cavities, although the two systems are of a fundamental different nature since we deal here with a Hamiltonian system whereas optical cavities are inherently dissipative systems. The generation of the sporadic bursts of light in regime (iii) is no longer related to coherent and deterministic quasi-soliton structures. This is corroborated by the statistics of the field, which approaches a Gaussian statistics, as expected for a fully incoherent system of weakly nonlinear waves [22,41-48]. Note however that a slight deviation from Gaussian statistics can be identified in the far-tail of the PDF (Fig. 4(a2)), a feature that may be precisely ascribed to the existence of extreme events. This aspect is also confirmed by the PDF of the intensity maxima (inset of Fig. 4(a2)), whose asymmetric shape has an origin analogous to that discussed in regime (ii). We remark that some extreme events in the far-tail of the PDFs (insets of Fig. 3b and 4(a2)) verify the usual hydrodynamic criterion [7], which defines a rogue wave as one exhibiting a maximal intensity higher than twice the average intensity among one third of the highest waves in the PDF of intensity maxima. Finally, for higher values of $\mathrm{H}(\overline{\mathrm{H}} \sim$ 30), the system becomes essentially 'linear', i.e. $\left|\mathrm{H}_{\mathrm{NL}}\right| \ll\left|\mathrm{H}_{\mathrm{L}}\right|$. We have verified that in this regime the PDF of the intensity maxima still exhibits an asymmetric shape (see Fig. 4b), which is almost indistinguishable from that obtained in the purely linear regime, i.e. without Kerr effect $(\gamma=0)$. 
We underline that the three regimes discussed here for $\sigma=0.02$ have been also identified for different values of the TOD coefficient, i.e. $\sigma=0.03$ and $\sigma=0.06$ (see Fig. 1b). The corresponding 'condensation curves' exhibit properties similar to that of $\sigma=0.02$. As expected, the average amplitude of the quasi-soliton in the coherent regime (i) decreases as the TOD coefficient $\sigma$ increases. Conversely, let us remark that the threshold value for the energy, $\overline{\mathrm{H}}_{\mathrm{c}}$, does not depend significantly on $\sigma$.

In conclusion, we provided some general physical insights into the spontaneous emergence of extreme events from turbulent fluctuations. The new optical rogue waves reported in the regime (ii) and, more importantly in regime (iii), seem to exhibit properties analogous to genuine hydrodynamic rogue wave events, a feature that needs to be analyzed in more detail so as to draw a substantiated analogy between them.

Acknowledgments: This research was supported by the Agence Nationale de la Recherche (COSTUME project, ANR-08-SYSC-004-03; MANUREVA project, ANR-08-SYSC019). 


\section{References}

1. D.R. Solli, C. Ropers, P. Koonath, B. Jalali, Nature 450 (2007) 1054-1057.

2. M.N. Islam, G. Sucha, I. Bar-Joseph, M. Wegener, J.P. Gordon, D.S. Chemla, J. Opt. Soc. Am. B 6 (1989) 1149-1158.

3. B.A. Malomed, Phys. Rev. A 44 (1991) 1412-1414.

4. M.H. Frosz, O. Bang, A. Bjarklev, Opt. Express 14 (2006) 9391-9407.

5. F. Luan, D.V. Skryabin, A.V. Yulin, J.C. Knight, Opt. Express 14 (2006) 9844-9853.

6. J.M. Dudley, G. Genty, S. Coen, Rev. Mod. Phys. 78 (2006) 1135-1184.

7. C. Kharif, E. Pelinovsky, A. Slunyaev, Rogue Waves in the Ocean, Springer-Verlag, Heidelberg, 2009.

8. J.M. Dudley, G. Genty, B.J. Eggleton, Opt. Express 16 (2008) 3644-3651.

9. G. Genty, J.M. Dudley, B.J. Eggleton, Appl. Phys. B 94 (2009) 187-194.

10. K. Hammani, C. Finot, G. Millot, Opt. Lett. 34 (2009) 1138-1140.

11. K. Hammani, C. Finot, B. Kibler, G. Millot, IEEE Phot. Journal 1 (2009) 205-212.

12. B. Kibler, C. Finot, J.M. Dudley, Eur. Phys. J. Special Topics 173 (2009) 289-295.

13. A. Mussot, A. Kudlinski, M. Kolobov, E. Louvergneaux, M. Douay, M. Taki, Opt. Express 17 (2009) 17010-17015.

14. N. Akhmediev, A. Ankiewicz, M. Taki, Phys. Lett. A 373 (2009) 675-678.

15. N. Akhmediev, J.M. Soto-Crespo, A. Ankiewicz, Phys. Lett. A 373 (2009) 2137-2145.

16. A. Ankiewicz, N. Akhmediev, Phys. Lett. A 373 (2009) 3997-4000.

17. N. Akhmediev, A. Ankiewicz, J.M. Soto-Crespo, Phys. Rev. E 80 (2009) 026601.

18. M. Taki, A. Mussot, A. Kudlinski, E. Louvergneaux, M. Kolobov, M. Douay, Phys. Lett. A 374 (2010) 691-695. 
19. G. Genty, C.M. de Sterke, O. Bang, F. Dias, N. Akhmediev, J.M. Dudley, Phys. Lett. A 374 (2010) 989-996.

20. J.M. Dudley, G. Genty, F. Dias, B. Kibler, N. Akhmediev, Opt. Express 17 (2009) 21497-21508.

21. V.E. Zakharov, N. Pushkarev, V.F. Shvets, V.V. Yan'kov, Pis'ma Zh. Eksp. Teor. Fiz. 48 (1988) 79 [JETP Lett. 48 (1988) 83].

22. V. Zakharov, F. Dias, A. Pushkarev, Phys. Reports 398 (2004) 1-65.

23. See, e.g., N. Akhmediev, M. Karlsson, Phys. Rev. A 51 (1995) 2602-2607.

24. R. Jordan, C. Josserand, Phys. Rev. E 61 (2000) 1527.

25. T. Dauxois, M. Peyrard, Phys. Rev. Lett. 70 (1993) 3935.

26. T. Cretegny, T. Dauxois, S. Ruffo, A. Torcini, Physica D 121 (1998) 109.

27. O. Bang and M. Peyrard, Physica D 81 (1995) 9.

28. O. Bang, M. Peyrard, Phys. Rev. E 53 (1996) 4143.

29. K. Rasmussen, T. Cretegny, P.G. Kevrekidis, N. Grønbech-Jensen, Phys. Rev. Lett. 84 (2000) 3740.

30. The Fermi-Pasta-Ulam Problem: A Status Report, edited by G. Gallavotti, Lecture Notes in Physics (Springer, New York, 2007).

31. B. Rumpf, A.C. Newell, Phys. Rev. Lett. 87 (2001) 054102.

32. B. Rumpf and A.C. Newell, Physica D 184 (2003) 162.

33. B. Barviau, B. Kibler, S. Coen, A. Picozzi, Opt. Lett. 33 (2008) 2833-2835.

34. B. Barviau, B. Kibler, A. Kudlinski, A. Mussot, G. Millot, A. Picozzi, Opt. Express 17 (2009) 7392.

35. B. Barviau, B. Kibler, A. Picozzi, Phys. Rev. A 79 (2009) 063840.

36. M.J. Davis, S.A. Morgan, K. Burnett, Phys. Rev. Lett. 87 (2001) 160402.

37. C. Connaughton, C. Josserand, A. Picozzi, Y. Pomeau, S. Rica, Phys. Rev. Lett. 95, (2005) 263901. 
38. G. Düring, A. Picozzi, S. Rica, Physica D 238 (2009) 1524-1549.

39. In statistical mechanics, the energy $\mathrm{H}$ usually provides a measure of the amount of excitation' in the physical system. In the weakly nonlinear regime, the energy per particle $\mathrm{H} / \mathrm{N}$ may also provide a rigorous measure of the amount of incoherence of a wave system, in analogy with kinetic gas theory, see e.g, A. Picozzi and S. Rica, Europhys. Lett. 84 (2008) 34004.

40. Note that Eq.(1) does not exhibit a conventional thermalization process, see P. Suret, S. Randoux, H. Jauslin and A. Picozzi, Phys. Rev. Lett. 104 (2010) 054101.

41. S. Dyachenko, A.C. Newell, A. Pushkarev, V.E. Zakharov, Physica D 57 (1992) 96-160.

42. V. Zakharov, V. L'vov, G. Falkovich, Kolmogorov Spectra of Turbulence I, Springer, Berlin, 1992.

43. A. Picozzi, Opt. Express 15 (2007) 9063-9083.

44. S. Pitois, S. Lagrange, H.R. Jauslin, and A. Picozzi, Phys. Rev. Lett. 97 (2006) 033902.

45. S. Lagrange, H.R. Jauslin, A. Picozzi, Europhys. Lett. 79 (2007) 64001.

46. S.A. Babin, D.V. Churkin, A.E. Ismagulov, S.I. Kablukov, E.V. Podivilov, J. Opt. Soc. Am. B 24 (2007) 1729-1738.

47. A. Picozzi, Opt. Express 16 (2008) 17171-17185.

48. U. Bortolozzo, J. Laurie, S. Nazarenko, S. Residori, J. Opt. Soc. Am. B 26 (2009) 22802284.

49. A. Montina, U. Bortolozzo, S. Residori, F.T. Arecchi, Phys. Rev. Lett. 103 (2009) 173901. 


\section{Figure caption:}

Fig. 1: Average of the maximum intensity peak detected in the temporal window during the last stage of propagation $\left(500 \mathrm{~L}_{0}\right)$ as a function of the Hamiltonian density $\overline{\mathrm{H}}=\mathrm{H} / \mathrm{T}$, (a) for $\sigma=0.02$, (b) for $\sigma=0.03$ (blue squares) and $\sigma=0.06$ (gray diamonds). The green dots (or diamonds) in (a) are analyzed separately through Figs. 2-4. All numerical simulations have been realized with the same power $\mathrm{N}$ (in normalized units $\mathrm{N} / \mathrm{T}=1$, where $\mathrm{T}=20$ is the size of the temporal window used in the simulations. A grid of 2048 points has been used).

Fig. 2: Regime (i): (a) Space-time intensity pattern showing a coherent quasi-soliton propagating in the midst of small-scale fluctuations for $\overline{\mathrm{H}}=-10.3(\varepsilon=2.85)$. (b) PDF of the intensity of the field and of the maxima of the intensity (inset). The gray dashed line stands for Gaussian statistics of the field amplitude. (c,d) Temporal intensity profiles corresponding to the dashed lines in (a).

Fig. 3: Regime (ii): (a) Space-time intensity pattern showing an intermittent quasi-soliton propagating in the midst of turbulent fluctuations for $\overline{\mathrm{H}}=13.3(\varepsilon=0.075)$. (b) PDF of the intensity of the field and of the maxima of the intensity (inset). The gray dashed line stands for Gaussian statistics of the field amplitude. (c,d) Temporal intensity profiles corresponding to the dashed lines in (a).

Fig. 4: Regime (iii): (a1,b1) Space-time intensity patterns for $\overline{\mathrm{H}}=19.5(\varepsilon=0.05)$ and $\overline{\mathrm{H}}=35.5$ $(\varepsilon=0.027) .(a 2, b 2)$ Corresponding PDF of the field intensity and of the maxima of the intensity (insets). The gray dashed line stands for Gaussian statistics of the field amplitude. (a3,a4) Temporal intensity profiles corresponding to the arrows in (a1). 


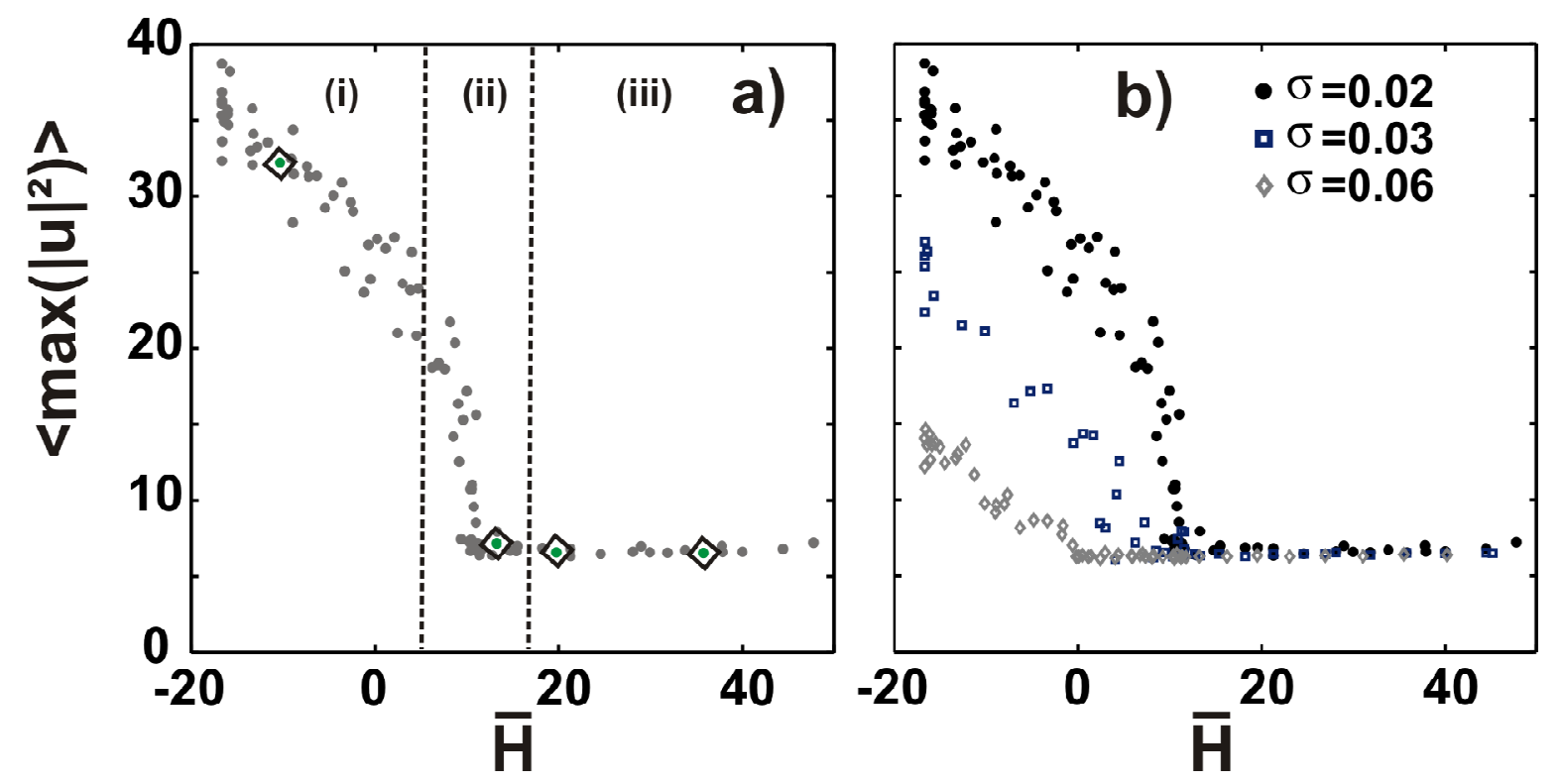

FIGURE 1 

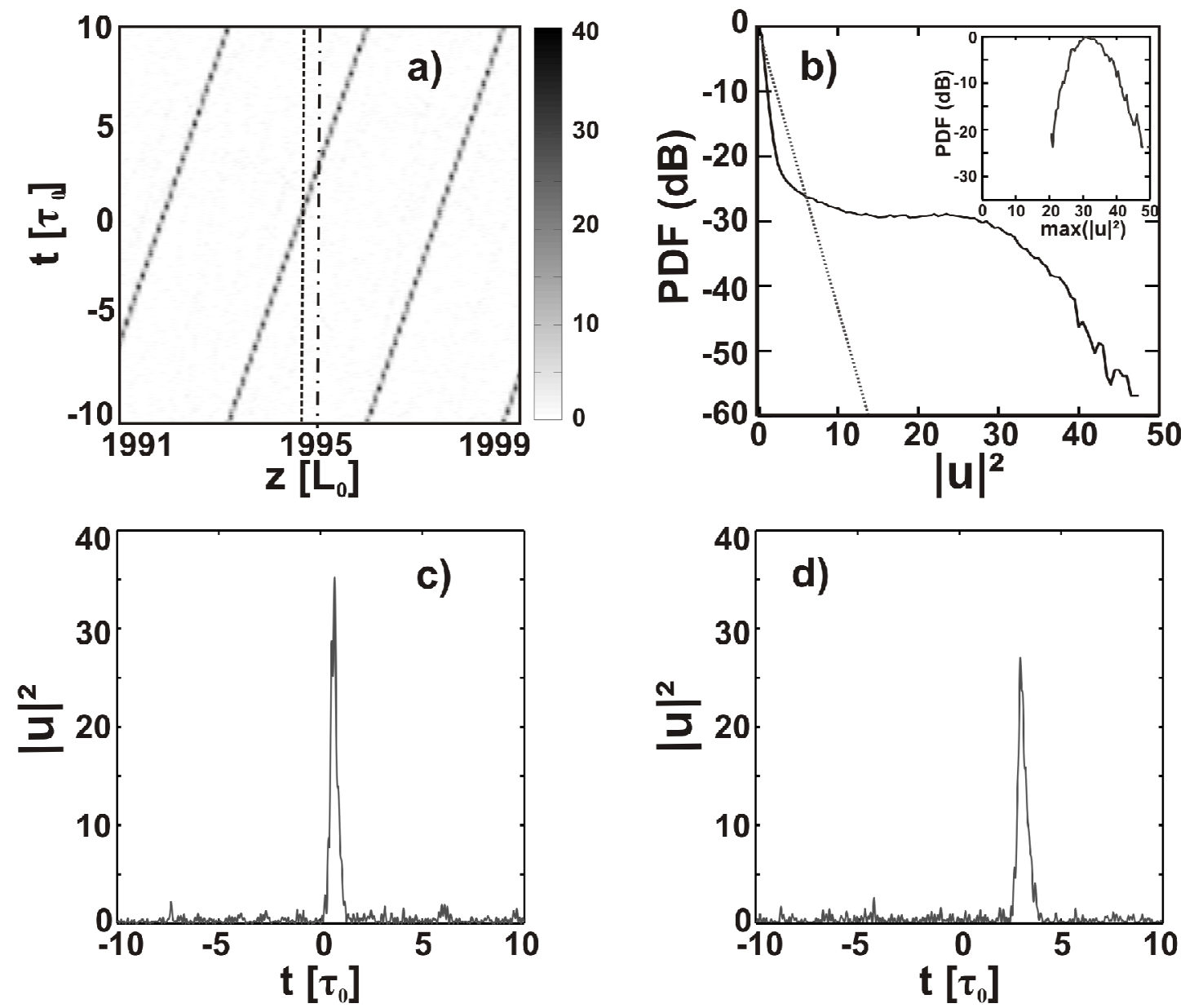

FIGURE 2 

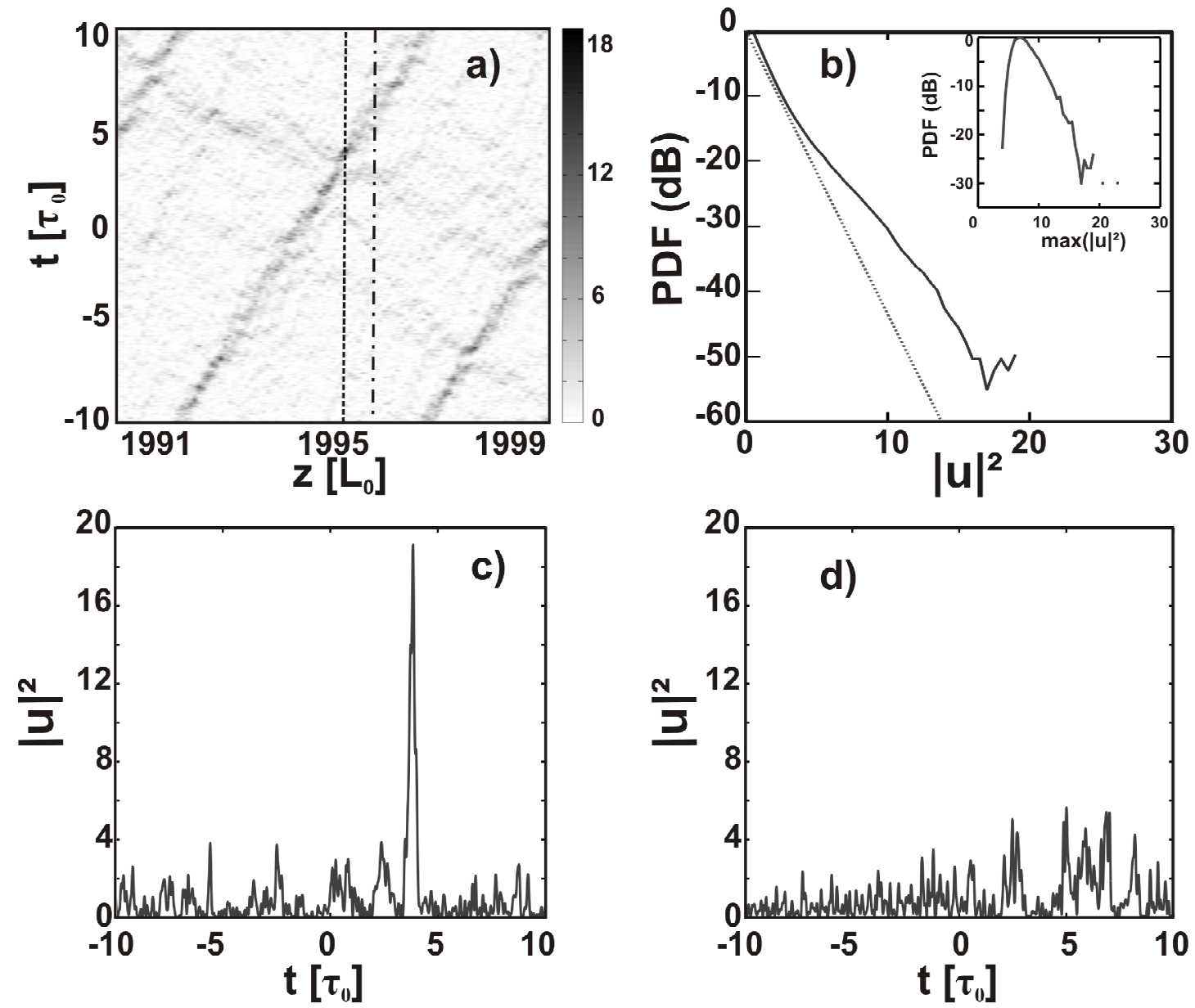

FIGURE 3 

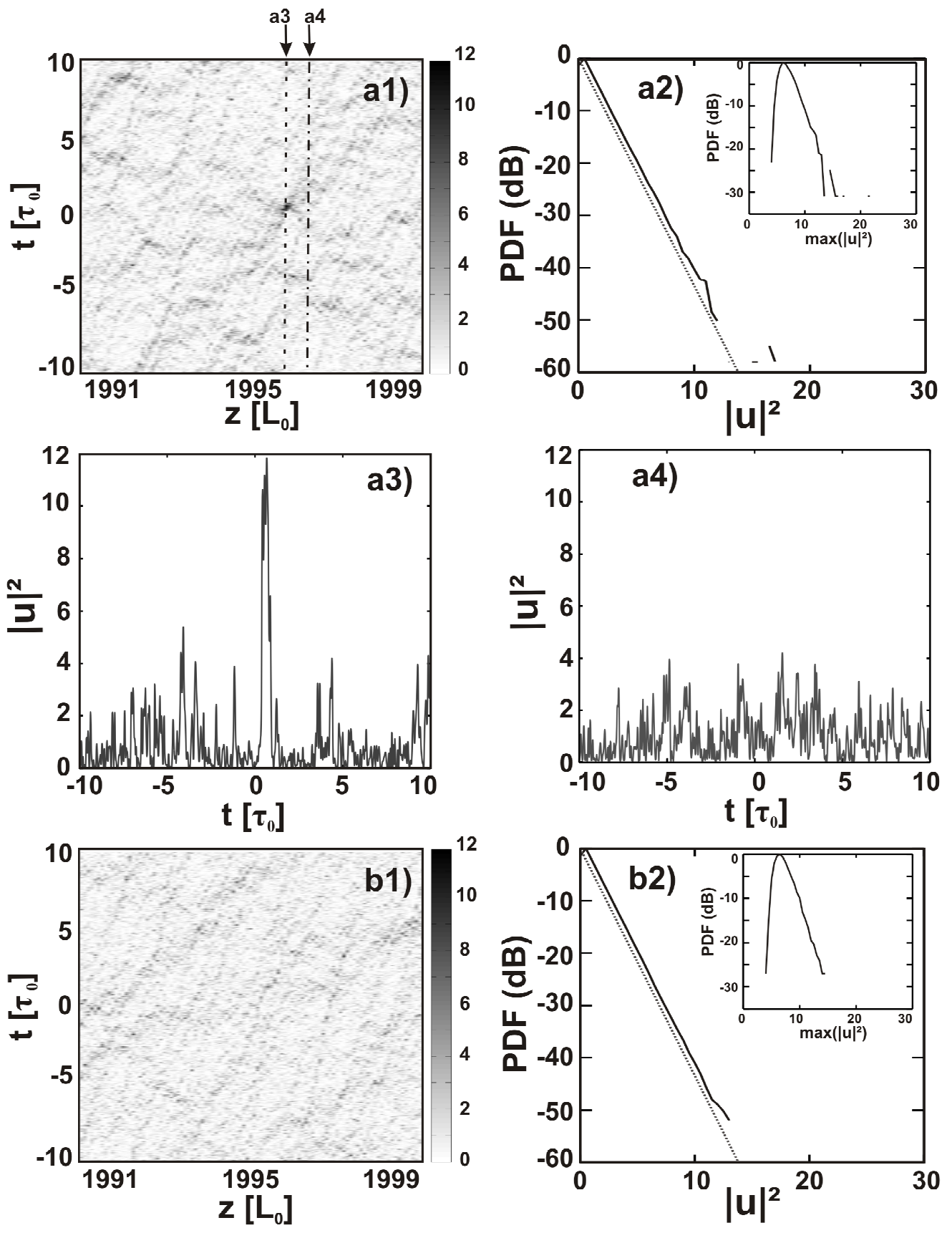

FIGURE 4 